PROCEEDINGS OF THE

AMERICAN MATHEMATICAL SOCIETY

Volume 126, Number 4, April 1998, Pages 1063-1069

S 0002-9939(98)04243-9

\title{
CHARACTERIZATIONS OF CONTRACTION $C$-SEMIGROUPS
}

\author{
MIAO LI AND FALUN HUANG
}

(Communicated by Palle E. T. Jorgensen)

\begin{abstract}
A $C$-semigroup $\{T(t)\}_{t>0}$ is of contractions if $\|T(t) x\| \leq\|C x\|$ for $t \geq 0, x \in X$. Using the Hille-Yosida space, we completely characterize the generators of contraction $C$-semigroups. We also give the Lumer-Phillips theorem for $C$-semigroups in several special cases.
\end{abstract}

\section{INTRODUCTION}

The notion of exponentially bounded $C$-semigroup was introduced by Davies and Pang [1]. Recently, the theory of $C$-semigroup has been extensively developed by many authors $[2,7,9]$. This theory allows us to study many ill-posed abstract Cauchy problems.

The starting point of this paper is to try to give an answer to the question asked by R. deLaubenfels in [3, Open question 6.10]: Does there exist an analogue of the Lumer-Phillips theorem for $C$-semigroups? Since the Lumer-Phillips theorem characterizes the generators of contraction $C_{0}$-semigroups, this gives us the motivation to make a suitable definition for the contractions of $C$-semigroups and then characterize the generators.

On the other hand, many works have generalized the Hille-Yosida theorem to $C$-semigroups. Earlier, Davies and Pang [1] gave a chracterization of an exponentially bounded $C$-semigroup under the assumption that $R(C)$ is dense in $X$. Later, Tanaka and Miyadera [7] generalized their results to the case of $R(C)$ not dense, and they gave a sufficient and necessary condition for a closed linear operator with dense domain to be the generator of an exponentially bounded $C$-semigroup. After defining the contraction $C$-semigroup, we are also interested in characterizing the generator by the Hille-Yosida type theorem. Here the main difficulty we meet with is that the generator may not be densely defined, we choose the Hille-Yosida space to give an additional condition on the generator.

This paper is organized as follows. $\S 2$ is devoted to some preliminaries on $C$ semigroups. In $\S 3$ we characterize the generators of contraction $C$-semigroups in general cases, and under the assumption that $C(D(A))$ is dense in $R(C)$, the characterization can be simplified. $\S 4$ deals with several special cases of $\rho(A) \neq \varnothing$ or

Received by the editors June 13, 1996 and, in revised form, September 23, 1996.

1991 Mathematics Subject Classification. Primary 47D03.

Key words and phrases. $C$-semigroups, $C_{0}$-semigroups, contraction, dissipative.

This project was supported by the National Science Foundation of China.

(C)1998 American Mathematical Society 
$\overline{R(C)}=X$, we obtain both the Hille-Yosida theorem and the Lumer-Phillips theorem in such cases. This means that we partly give the answer to the question mentioned above in the affirmative.

\section{Preliminaries}

Throughout this paper, $X$ will be a Banach space. The space of all bounded linear operators on $X$ will be denoted by $B(X)$, and $C$ will always be an injective operator in $B(X)$. For an operator $A$, we will write $D(A)$ for its domain, $R(A)$ for its range and $\rho(A)$ for its resolvent set, and we will write $\bar{E}$ for the closure of a subspace of $X, E$.

First, we recall the definition of $C$-semigroups.

Definition 2.1. A strongly continuous family $\{T(t)\}_{t \geq 0} \subset B(X)$ is called a $C$ semigroup if $T(t+s) C=T(t) T(s)$ for $t, s \geq 0$ and $T(0)=C .\{T(t)\}_{t>0}$ is exponentially bounded if there exist $M<\infty$ and $\omega \in R$ such that $\|T(t)\| \leq M e^{\omega t}$.

The generator of $\{T(t)\}_{t \geq 0}, A$, is defined by

$$
A x=C^{-1}\left[\lim _{t \downarrow 0} \frac{1}{t}(T(t) x-C x)\right]
$$

with

$$
D(A)=\left\{x \in X: \lim _{t \downarrow 0} \frac{1}{t}(T(t) x-C x) \text { exists and is in } R(C)\right\} .
$$

The complex number $\lambda$ is in $\rho_{C}(A)$, the $C$-resolvent of $A$, if $(\lambda-A)$ is injective and $R(C) \subseteq R(\lambda-A)$.

Lemma $2.2([4,7])$. Suppose $A$ generates a C-semigroup $\{T(t)\}_{t \geq 0}$ satisfying $\|T(t)\| \leq M e^{\omega t}$. Then

(a) $A$ is a closed linear operator with $\overline{D(A)} \supseteq R(C)$;

(b) $\forall x \in X, T(t) x=C x+A \int_{0}^{t} T(s) x d s$, which implies $T(\cdot) x$ is a mild solution for the abstract Cauchy problem

$$
\frac{d}{d t} u(t)=A u(t), \quad u(0)=x
$$

(c) $\forall x \in D(A)$ and $t \geq 0, T(t) x \in D(A)$ with $A T(t) x=T(t) A x$;

(d) $A=C^{-1} A C$, where $D\left(C^{-1} A C\right)=\{x \in X: C x \in D(A)$ and $A C x \in R(C)\}$;

(c) $(\omega, \infty) \subseteq \rho_{C}(A)$. For every $r>\omega$ and $n \in \mathbf{N}, D\left((r-A)^{-n}\right) \supseteq R(C)$ and

$$
(r-A)^{-n} C=\frac{1}{(n-1) !} \int_{0}^{\infty} t^{n-1} e^{-r t} T(t) d t
$$

which implies $\left\|(r-\omega)^{n}(r-A)^{-n} C\right\| \leq M$.

Next we need to introduce the Hille-Yosida space for an operator, for the details we refer to [4].

Definition 2.3. Suppose $A$ has no eigenvalues in $(0, \infty)$. The Hille-Yosida space for $A, Z_{0}$, is the Banach space defined by

$$
\begin{array}{r}
Z_{0}=\{x \in X: \text { The Cauchy problem (1) has a bounded uniformly } \\
\text { continuous mild solution } u(\cdot, x)\}
\end{array}
$$


with

$$
\|x\|_{Z_{0}}=\sup \{\|u(t, x)\| ; t \geq 0\} \quad \text { for } x \in Z_{0} .
$$

And the weak Hille-Yosida space for $A, Y$, is the Banach space defined by

$$
\begin{aligned}
Y=\left\{x \in X: x \in R\left((s-A)^{n}\right)\right. & \forall s>0, n \in \mathbf{N} \text { with } \\
\|x\|_{Y} & \left.=\sup \left\{s^{n}\left\|(s-A)^{-n} x\right\| ; s>0, n+1 \in \mathbf{N}\right\}<\infty\right\} .
\end{aligned}
$$

The relation between $Z_{0}$ and $Y$ is as follows.

Lemma 2.4. Suppose $A$ has no eigenvalues in $(0, \infty)$, and $Z_{0}$ and $Y$ are defined as above. Then

(a) $Z_{0} \subset Y$ and $\|x\|_{Z_{0}}=\|x\|_{Y}$ for all $x \in X$;

(b) $Z_{0}$ is the closure, in $Y$, of $D\left(\left.A\right|_{Y}\right)$, where $D\left(\left.A\right|_{Y}\right)=\{x \in Y \cap D(A): A x \in$ $Y\}$;

(c) $\left.A\right|_{Z_{0}}$ generates a contraction $C_{0}$-semigroup on $Z_{0}$.

\section{Characterizations of Contraction $C$-Semigroups}

A $C$-semigroup $\{T(t)\}_{t \geq 0}$ is of contractions if $\|T(t) x\| \leq\|C x\|$ for $t \geq 0$ and $x \in X$. In this section, we give the characterizations of the generators of contraction $C$-semigroups. We start with the following

Proposition 3.1. Suppose $A$ generates a contraction $C$-semigroup, then

(a) $(0, \infty) \subseteq \rho_{C}(A)$, and for $\lambda>0, n \in \mathbf{N}$ and $x \in X, R(C) \subseteq R\left((\lambda-A)^{n}\right)$ with

$$
\lambda^{n}\left\|(\lambda-A)^{-n} C x\right\| \leq\|C x\|
$$

(b) for every $x \in D(A)$, there exists an $x^{*} \in F(C x)$, that is, $x^{*} \in X^{*},\left\|x^{*}\right\|=$ $\|C x\|$ and $x^{*}(C x)=\|C x\|^{2}$, such that

$$
\operatorname{Re}\left\langle C A x, x^{*}\right\rangle \leq 0,
$$

where $\left\langle x, x^{*}\right\rangle$ denotes the value of $x^{*}$ at $x$.

Proof. (a) follows directly from Lemma $2.2(e)$.

Let $x \in D(A)$ and $x^{*} \in F(C x)$. Then

$$
\operatorname{Re}\left\langle\frac{T(t) x-C x}{t}, x^{*}\right\rangle \leq R e\left\langle\frac{T(t) x}{t}, x^{*}\right\rangle-\frac{\|C x\|^{2}}{t} \leq 0 \quad \text { for } t>0,
$$

hence

$$
R e\left\langle C A x, x^{*}\right\rangle=\lim _{t \downarrow 0} \operatorname{Re}\left\langle\frac{T(t) x-C x}{t}, x^{*}\right\rangle \leq 0
$$

This is (b).

Remark 3.2. If an operator $A$ with $C A \subseteq A C$ satisfies (b), we call $A C$-dissipative. Similar to the proof of [5, Chapter 1 , Theorem 4.2], we can prove that $A$ is $C$ dissipative if and only if $\|(\lambda-A) C x\| \geq \lambda\|C x\| \forall x \in D(A)$ and $\lambda>0$. Note that if $\lambda\left\|(\lambda-A)^{-1} C x\right\| \leq\|C x\|$ for all $x \in X$, then for $x \in D(A)$,

$$
\|(\lambda-A) C x\|=\|C(\lambda-A) x\| \geq\left\|\lambda(\lambda-A)^{-1} C(\lambda-A) x\right\|=\lambda\|C x\| .
$$

Using the Hille-Yosida space, we can completely characterize the generators.

Theorem 3.3. Let $A$ be an operator on $X$. Then $A$ generates a contraction $C$ semigroup if and only if $A$ satisfies 
(a) $A=C^{-1} A C$;

(b) $(0, \infty) \subseteq \rho_{C}(A), R(C) \subseteq R\left((\lambda-A)^{n}\right)$ and $\lambda^{n}\left\|(\lambda-A)^{-n} C x\right\| \leq\|C x\|$ for $\lambda>0, n \in \mathbf{N}$ and $x \in X$

(c) for some $\lambda \geq 0$, the Hille-Yosida space for $A-\lambda I$, denoted by $Z_{\lambda}$, contains $R(C)$.

Proof. For the necessity, Lemma 2.2(d) and Proposition 3.1 imply (a) and (b). It remains to show (c). Let $\lambda>0$ and define $S(t)=e^{-\lambda t} T(t)$ for $t \geq 0$. Thus $\{S(t)\}_{t \geq 0}$ is a bounded uniformly strongly continuous $C$-semigroup, generated by $A-\lambda I$. By Lemma 2.2(b) and Definition 2.3, $R(C)$ is contained in the Hille-Yosida space for $A-\lambda I$, i.e., $Z_{\lambda}$.

Conversely, let $A_{\lambda}=\left.A\right|_{Z}$. By Lemma 2.4, $A_{\lambda}-\lambda I$ generates a $C_{0^{-}}$-semigroup of contractions, $e^{t\left(A_{\lambda}-\lambda I\right)}$, on $\left(Z_{\lambda},\|\cdot\|_{Z_{\lambda}}\right)$, which implies $e^{t A_{\lambda}}$ is also a $C_{0}$-semigroup on $\left(Z_{\lambda}\|\cdot\|_{Z_{\lambda}}\right)$.

For $t \geq 0$, define $W(t): X \rightarrow X$ by $W(t)=e^{t A_{\lambda}} C$; we show that $\{W(t)\}_{t \geq 0}$ is a $C$-semigroup generated by $A$.

In fact, by (a), $C A \subseteq A C$, so that $C$ commutes with $e^{t A_{\lambda}}$ for $t \geq 0$. Thus

$$
W(t+s) C x=e^{(t+s) A_{\lambda}} C^{2} x=e^{t A_{\lambda}} C e^{s A_{\lambda}} C x=W(t) W(s) x,
$$

that is, $W(t+s) C=W(t) W(s)$.

Moreover, if $x \in D(A)$, then $C x \in Z_{\lambda} \cap D(A)$ with $A C x=C A x \in Z_{\lambda}$, so that $C x \in D\left(A_{\lambda}\right)$, which implies that $e^{t A_{\lambda}} C x$ is differentiable and

$$
A e^{t A_{\lambda}} C x=A_{\lambda} e^{t A_{\lambda}} C x=e^{t A_{\lambda}} A_{\lambda} C x=e^{t A_{\lambda}} C A x,
$$

hence $W(t) x \in D(A)$ with $A W(t) x=W(t) A x$. So $W(t)$ is generated by an extension of $A$. To show $A$ is the generator, we only need to prove that $A$ is closed. It is exactly as in the proof of [9, Lemma 2.2].

Finally, by (b) and the exponential formulas for $C$-semigroups, we have

$$
\|W(t) x\|=\lim _{n \rightarrow \infty}\left\|\left(1-\frac{t}{n} A\right)^{-n} C x\right\| \leq\|C x\|,
$$

so that $\{W(t)\}$ is of contractions.

Condition (c) in Theorem 3.3 seems to be difficult to check, but in the case of $C(D(A))$ dense in $R(C)$, it can be omitted.

Theorem 3.4. Suppose $C(D(A))$ is dense in $R(C)$. Then A generates a contraction $C$-semigroup if and only if $A$ satisfies (a) and (b) in Theorem 3.3.

Proof. We only need to show the sufficiency.

If $x \in D(A)$, then $C x \in D(A)$ with $A C x=C A x$ by (a). By (b), $R(C) \subseteq Y$, the weak Hille-Yosida space for $A$, since $Z_{0}$ is the closure of $D\left(\left.A\right|_{Y}\right)$ in $Y$ by Lemma 2.4, we have $C x \in Z_{0}$.

For all $x \in X$, there exists a sequence $\left\{x_{n}\right\} \subset D(A)$, such that $C x_{n} \rightarrow C x$, in $X$. Moreover, for $n, m \in \mathbf{N}$, by Lemma 2.4 ,

$$
\left\|C x_{n}-C x_{m}\right\|_{Z_{0}}=\left\|C x_{n}-C x_{m}\right\|_{Y} \leq\left\|C x_{n}-C x_{m}\right\|,
$$

so that $\left\{C x_{n}\right\}$ is a Cauchy sequence in $Z_{0}$, which implies $R(C) \subseteq Z_{0}$. So Theorem 3.4 follows from Theorem 3.3. 
From the proof above we know that in this case we can choose $\lambda=0$ in Theorem $3.3(\mathrm{c})$.

Note that if $D(A)$ is dense in $X$, then $C(D(A))$ is dense in $R(C)$. However, [2, Example 6.2] gave an example of a $C$-semigroup whose generator $A$ is not densely defined while $C(D(A))$ is dense in $R(C)$.

\section{Special CASES}

In this section, we make some applications of the results from the preceding section. First we give a sufficient condition that $C(D(A))$ is dense in $R(C)$.

Lemma 4.1. Suppose that $A$ generates an exponentially bounded $C$-semigroup and there exists a sequence $\left\{\lambda_{n}\right\} \subset \rho(A)$, such that $\lambda_{n} \rightarrow+\infty$, then $C(D(A))$ is dense in $R(C)$.

Proof. Let $\lambda \in \rho(A)$, then for $\forall x \in X,(\lambda-A)^{-1} C x=C(\lambda-A)^{-1} x \in C(D(A))$. An estimation using Eq. (2) yields that $\lambda(\lambda-A)^{-1} C x \rightarrow C x$ as $\lambda \rightarrow+\infty$. So that $\lambda_{n}\left(\lambda_{n}-A\right)^{-1} C x \rightarrow C x(n \rightarrow \infty)$, and since $\lambda_{n}\left(\lambda_{n}-A\right)^{-1} C x \in C(D(A))$, our result holds.

The next lemma will be needed in the sequel.

Lemma 4.2. Let $A$ be a closed linear operator with $C A \subseteq A C$. Suppose $0 \neq \lambda \in$ $\rho_{C}(A)$ and $\lambda\left\|(\lambda-A)^{-1} C x\right\| \leq\|C x\|, \forall x \in X$. Then $R(\lambda-A) \supseteq \overline{R(C)}$, and $\lambda\left\|(\lambda-A)^{-1} x\right\| \leq\|x\|$ for all $x \in \overline{R(C)}$.

Proof. Let $x \in \overline{R(C)}$. There exists a sequence $\left\{x_{n}\right\} \subset X$ such that $C x_{n} \rightarrow x$ as $n \rightarrow \infty$. Define $x_{n}^{\prime}=(\lambda-A)^{-1} C x_{n}$, thus

$$
\left\|x_{n}^{\prime}-x_{m}^{\prime}\right\|=\left\|(\lambda-A)^{-1} C\left(x_{n}-x_{m}\right)\right\| \leq \frac{1}{\lambda}\left\|C\left(x_{n}-x_{m}\right)\right\|
$$

for $n, m \in \mathbf{N}$, so $\left\{x_{n}^{\prime}\right\}$ is a Cauchy sequence. Suppose $x_{n}^{\prime} \rightarrow x_{0} \in X$ as $n \rightarrow \infty$. Since $(\lambda-A) x_{n}^{\prime}=C x_{n}$ and $A$ is closed, it follows that $x_{0} \in D(A)$ and $(\lambda-A) x_{0}=x$. Moreover,

$$
\begin{aligned}
\lambda\left\|(\lambda-A)^{-1} x\right\|=\lambda\left\|x_{0}\right\|=\lim _{n \rightarrow \infty}\left\|x_{n}^{\prime}\right\| & =\lim _{n \rightarrow \infty} \lambda\left\|(\lambda-A)^{-1} C x_{n}\right\| \\
& \leq \lim _{n \rightarrow \infty}\left\|C x_{n}\right\|=\|x\|,
\end{aligned}
$$

as desired.

Now we can apply Theorem 3.4 to the case of $\rho(A) \neq \varnothing$. It is remarked that since $\rho(A) \neq \varnothing, C A \subseteq A C$ implies $A=C^{-1} A C$.

Theorem 4.3. Let $A$ be an operator on $X$. Suppose that $(0, \infty) \subseteq \rho(A)$. Then $A$ generates a contraction $C$-semigroup if and only if $A$ satisfies

(a) $C A \subseteq A C$;

(b) $\lambda\left\|(\lambda-A)^{-1} C x\right\| \leq\|C x\|$ for $\lambda>0$ and $x \in X$;

(c) $C(D(A))$ is dense in $R(C)$.

Proof. Theorem 3.3 and Lemma 4.2 imply the necessity.

Conversely, we define an operator $B$ on $X$ by

$$
D(B)=\{C x: x \in D(A)\}, B x=A x \quad \text { for } x \in D(B) .
$$

So that $D(B)=C(D(A))$ and $R(B) \subseteq R(C)$. For $\lambda>0$, since $(\lambda-A)^{-1} C x=$ $C(\lambda-A)^{-1} x$, so $R(\lambda-B) \supseteq R(C)$ and $\lambda\left\|(\lambda-B)^{-1} C x\right\|=\lambda\left\|(\lambda-A)^{-1} C x\right\| \leq$ 
$\|C x\|$. From Remark 3.2, we know that $B$ is dissipative on $(\overline{R(C)},\|\cdot\|)$. Since $\overline{D(B)}=C(D(A))=\overline{R(C)}$, by [5, Chapter 1 , Theorem 4.3], $B$ is closable in $\overline{R(C)}$ (hence in $X$ ), and the closure of $B$ in $(\overline{R(C)},\|\cdot\|$ ) (or $X), \bar{B}$, is dissipative on $(\overline{R(C)}$, $\|\cdot\|)$. By Lemma 4.2, $R(\lambda-\bar{B})=\overline{R(C)}$ for $\lambda>0$. Therefore, the Lumer-Phillips theorem for $C_{0}$-semigroups implies that $\bar{B}$ generates a contraction $C_{0}$-semigroup, $\{S(t)\}_{t \geq 0}$, on $(\overline{R(C)},\|\cdot\|)$. Define $T(t): X \rightarrow X$ by $T(t)=S(t) C$. Thus $\{T(t)\}_{t \geq 0}$ is a $C$-semigroup of contractions on $X$. For $x \in D(A)$,

$$
\frac{T(t) x-C x}{t}=\frac{S(t) C x-C x}{t} \rightarrow B C x=C A x,
$$

so that an extension of $A$ is the generator, and since $\rho(A) \neq \varnothing$, it is exactly $A$.

Remark 4.4. (a) The conditions (a)-(c) in Theorem 4.3 are equivalent to (a), (c) and (b) $A$ is $C$-dissipative. In fact, by Remark 3.2, (b)' implies that $\|\lambda(\lambda-A) C x\|$ $\geq \lambda\|C x\|(\lambda>0, x \in D(A))$. Since $(0, \infty) \subseteq \rho(A)$, for $\lambda>0$,

$$
\|C x\|=\left\|(\lambda-A) C(\lambda-A)^{-1} x\right\| \geq \lambda\left\|C(\lambda-A)^{-1} x\right\|=\lambda\left\|(\lambda-A)^{-1} C x\right\|,
$$

which is (b).

(b) In [2, Theorem 3.3], it is claimed that if $\rho(A) \neq \varnothing$ and $A$ generates a $C$ semigroup of $O\left(e^{\omega t}\right)$, then $(\omega, \infty) \subset \rho(A)$. However, there appears to be a gap in the argument, because it fails to prove that, if $C^{-1}$ and $(r-A)$ both have resolvents that commute, then $C^{-1}(r-A)=(r-A) C^{-1}$. Here is a counterexample, suggested by deLaubenfels himself. Take $X=B C([0, \infty))$, the space of all bounded continuous functions on $[0, \infty)$ with supremum norm. Define $(A f)(s)=-s f(s)$ with $D(A)=\{f \in X, A f \in X\}$ and $(C f)(s)=\frac{s}{1+s} f(s)$ for $s \geq 0$. Then $\sigma(A)$ (the spectrum of $A)=(-\infty, 0]$, and $C^{-1}(\lambda-A)=(\lambda-A) C^{-1}$ for all $\lambda \in \rho(A)$. It is obvious that the function $f(s)=\frac{1}{1+s}$ is in $D\left(C^{-1} A\right)$ but is not in $D\left(A C^{-1}\right)$. Thus $C^{-1} A \neq A C^{-1}$. We do not know whether the claimed result remains true.

Let us consider the case when $\rho(A) \neq \varnothing$. Let $C=(r-A)^{-n}$, where $r \in \rho(A)$ and $n \in \mathbf{N} \cup\{0\}$. From [9, Lemma 6.1], we know $\rho_{C}(A)=\rho(A)$. Since $R(C)=D\left(A^{n}\right)$ and $C(D(A))=D\left(A^{n+1}\right)$, as a direct consequence of Theorem 4.3, we have

Corollary 4.5. Suppose $r \in \rho(A) \neq \varnothing$, let $C=(r-A)^{-n}, n \in \mathbf{N} \cup\{0\}$. Then the following statements are equivalent.

(a) A generates a contraction $C$-semigroup;

(b) A satisfies

(i) $(0, \infty) \subseteq \rho_{C}(A)$,

(ii) $\forall x \in D\left(A^{n}\right)$ and $\lambda>0,\left\|\lambda(\lambda-A)^{-1} x\right\| \leq\|x\|$,

(iii) $D\left(A^{n+1}\right)$ is dense in $D\left(A^{n}\right)$;

(c) A satisfies (i), (iii) and

(ii)' $A$ is $C$-dissipative.

In the case of $\overline{R(C)}=X$, the generator of a contraction $C$-semigroup is in fact the generator of a contraction $C_{0}$-semigroup.

Theorem 4.6. Suppose $\overline{R(C)}=X$. Then the following assertions are equivalent:

(a) A generates a contraction $C$-semigroup $\{T(t)\}_{t \geq 0}$;

(b) A generates a contraction $C_{0}$-semigroup $\{S(t)\}_{t \geq 0}$ and $C A \subseteq A C$;

(c) A satisfies

(i) $A$ is closed and $C A \subseteq A C$, 
(ii) $(0, \infty) \subseteq \rho_{C}(A)$ and $\lambda\left\|(\lambda-A)^{-1} C x\right\| \leq\|C x\|$ for $\lambda>0, x \in X$,

(iii) $D(A)$ is dense;

(d) A satisfies (i), (iii) and

(ii) $^{\prime}(0, \infty) \subseteq \rho_{C}(A)$ and $A$ is $C$-dissipative.

Proof. (a) $\Rightarrow(\mathrm{c})$ and $(\mathrm{c}) \Rightarrow(\mathrm{d})$ are obvious.

(c) $\Rightarrow$ (b). By Lemma 4.2, (ii) implies $R(\lambda-A)=X$ and $\lambda\left\|(\lambda-A)^{-1} x\right\| \leq\|x\|$ for $\lambda>0$ and $x \in X$, applying the Hille-Yosida theorem for $C_{0}$-semigroups to $A$ gives (b).

(b) $\Rightarrow\left(\right.$ a). By defining $T(t)=S(t) C$, it is not hard to show that $\{T(t)\}_{t \geq 0}$ is a contraction $C$-semigroup generated by $A$.

$(\mathrm{d}) \Rightarrow(\mathrm{c})$. Since $A$ is $C$-dissipative, $\forall x \in D(A)$, we have $\|(\lambda-A) C x\| \geq \lambda\|C x\|$, so that for $x \in R(\lambda-A)$,

$$
\|C x\|=\left\|(\lambda-A) C(\lambda-A)^{-1} x\right\| \geq \lambda\left\|C(\lambda-A)^{-1} x\right\|=\lambda\left\|(\lambda-A)^{-1} C x\right\| .
$$

Since $R(\lambda-A) \supseteq R(C)$, which is dense in $X$, a similar proof as that of Lemma 4.2 will do.

Consider when $B$ generates a contraction $C_{0}$-semigroup $\{S(t)\}_{t \geq 0}$ on $\overline{R(C)}$ and $C B \subseteq B C$. Define $T(t)=S(t) C(t \geq 0)$, we get a contraction $C$-semigroup $\{T(t)\}_{t \geq 0}$ on $X$. Suppose $A$ is the generator. It is not hard to verify that $B=$ $A \mid \overline{R(C)}$. For the converse, in the case of Theorem 4.3, we know it is true.

Open Question. Suppose $A$ is the generator of a contraction $C$-semigroup on a Banach space $X$. Does there exist a restriction of $A, A^{\prime}$, which is a generator of a contraction $C_{0}$-semigroup on $\overline{R(C)}$ ?

\section{REFERENCES}

1. E. B. Davies and M. M. Pang, The Cauchy problem and a generalization of the Hille-Yosida theorem, Proc. London Math. Soc. 55 (1987), 181-208. MR 88e:34100

2. R. deLaubenfels, C-semigroups and the Cauchy problem, J. Funct. Anal. 111 (1993), 44-61. MR 94b:47055

3. _ C-semigroups and strongly continuous semigroups, Israel J. Math. 81 (1993), 227255. MR 95d:47047

4. _ Existence families, functional calculi and evolution equations, Lecture Notes in Math., Vol. 1570, Springer Verlag, 1994. MR 96b: 47047

5. A. Pazy, Semigroups of linear operators and applications to partial differential equations, Springer-Verlag, New York, 1983. MR 85g:47061

6. N. Tanaka, On the exponentially bounded C-semigroups, Tokyo J. Math. 10 (1987), 107-117. MR 88h:47063

7. N. Tanaka and I. Miyadera, Exponentially bounded C-semigroups and integrated semigroups, Tokyo J. Math. 12 (1989), 99-115. MR 90g:47081

8. Exponentially bounded C-semigroups and generation of semigroups, J. Math. Anal. Appl. 143 (1989), 358-378. MR 90k:47087

9. Q. Zheng and Liping Liu, Almost periodic regularized groups, semigroups, and cosine functions, J. Math. Anal. Appl. 197 (1996), 90-162. MR 96m:47076

Department of Mathematics, Sichuan Union University, Chengdu 610064, People's Republic of China 